XXXI International Workshop on High Energy Physics:

Critical Points in the Modern Particle Physics

International Journal of Modern Physics: Conference Series

Vol. 47 (2018) 1860098 (6 pages)

(C) The Author(s)

DOI: $10.1142 / S 2010194518600984$

\title{
Measurements of Cross Sections and Couplings of the Higgs Boson Using the ATLAS Detector
}

\author{
B. Stugu (on behalf of the ATLAS Collaboration) \\ Department of Physics and Technology, \\ University of Bergen Bergen, Norway \\ Bjarne.Stugu@uib.no
}

Published 6 June 2018

\begin{abstract}
Measurements of cross sections and couplings of the Higgs boson using the ATLAS detector at CERN's LHC are presented. Data from proton proton collisions at $\sqrt{s}=7$, 8 and $13 \mathrm{TeV}$ are discussed. A range of production and decay couplings can be tested with a precision that depends on the generality of the assumptions made. Data at 7 and $8 \mathrm{TeV}$ are also combined with CMS results to enhance the precision. The results are all consistent with predictions of the Standard Model.
\end{abstract}

Keywords: Atlas; Higgs; Cross Section; Couplings.

\section{Introduction}

Following the discovery of a particle consistent with the Standard Model (SM) Higgs boson, ${ }^{1,2}$ it is of great interest to test its properties against the predictions of the SM. Any deviations would hint towards the existence of physics beyond the SM. Since the discovery in July 2012, the data sample has increased steadily in size and includes data from 2011 at $7 \mathrm{TeV}$, from 2012 at $8 \mathrm{TeV}$, as well as a large data set at $\sqrt{s}=13 \mathrm{TeV}$ collected in 2015 and 2016. The comparisons with SM predictions are possible with increased precision.

\section{Higgs production and decay modes}

The dominating production process of the Higgs at the LHC is through the fusion of two gluons in the collisions (Fig. 1 a), accounting for close to $90 \%$ of the cross section. The second process is through fusion of heavy vector bosons (VBF), and accounts for around $8 \%$ (Fig. 1 b). Despite the lower cross section, Higgs bosons in this process

This is an Open Access article published by World Scientific Publishing Company. It is distributed under the terms of the Creative Commons Attribution 4.0 (CC-BY) License. Further distribution of this work is permitted, provided the original work is properly cited. 


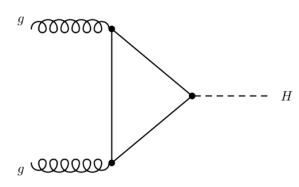

a)

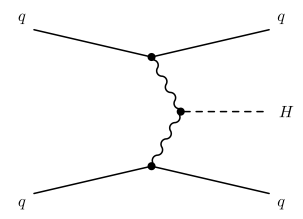

b)

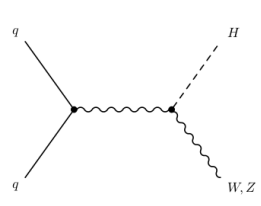

c)

Fig. 1. Production modes of the Higgs in proton-proton collisions at the LHC: a) gluon-gluon fusion, b) Vector boson fusion (VBF) and c) Vector boson-Higgs $(V H)$ production.

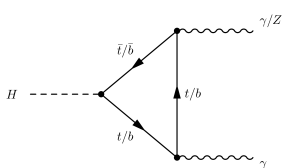

a)

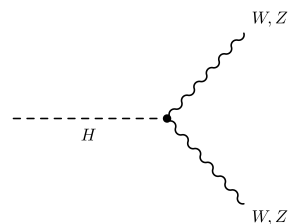

b)

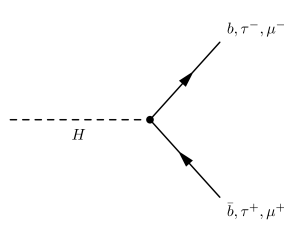

c)

Fig. 2. Decay modes of the Higgs boson: a) two photons, b) two vector bosons, c) two fermions.

form a significant part of selected event samples in some channels due to important additional handles that can be used in the selection process: two energetic quark jets emerging with high rapidity separation, and a Higgs boson emerging with high transverse momentum. With increasing data-samples, production together with a $W$ or $Z$ boson ( $V H$, Fig. 1 c) can also be identified and accounted for.

All Higgs coupling vertices include the mass of the particles involved, with a strength proportional to the mass of the produced fermion-antifermion or vector boson pair. The dominating tree-level decay mode is therefore a pair of $b$-quarks. Unfortunately, this decay mode has modest experimental mass resolution, and is extremely difficult to identify in the large background of multi-jet events produced at the LHC. The two most important processes for experimental identification of Higgs particles are decays to two photons and to a $Z Z^{*}$ pair where both bosons decay to a pair of electrons or muons. These decay channels both have permille level branching fractions. Figure 2 shows Feynman diagrams of the most important decay channels.

\section{Analysis Strategy}

\subsection{Cross Sections}

The number of experimentally observed events will always depend on the product $\sigma_{i} \times B R_{f}$, where $\sigma_{i}$ is the production cross section in a selected production mode $i$, and $B R_{f}$ is the decay branching fraction to final state $f$ observed. For the purpose of comparing with SM expectations, simulated event samples that are generated in accordance with SM expectations. These samples are taken through the full simulation of the ATLAS detector response and are reconstructed using the same 
procedure as for real data. The final efficiency and background estimates are derived using suitable samples of real data.

Comparisons with SM predictions are performed by forming the signal strength as defined by the ratio:

$$
\mu_{i}^{f}=\frac{\sigma_{i} \times B R_{f}}{\left(\sigma_{i} \times B R_{f}\right)_{\mathrm{SM}}}=\mu_{i} \times \mu_{f} .
$$

Signal strengths are measured individually in specific production and decay modes assuming SM expectations of the different Higgs decay branching fractions. Contributions due to different production modes can be derived separately and summed to get a single overall production signal strength.

\subsection{Couplings}

Since the Higgs boson is observed in several combinations of production and decay modes, the cross sections depend on individual couplings that appear in various combinations. The couplings are parametrized in terms of possible deviations from the SM, in the ' $\kappa$ framework' developed by the LHC Higgs working group: ${ }^{3}$

$$
\sigma(i \rightarrow H \rightarrow f)=\frac{\sigma_{i}\left(\kappa_{j}\right) \cdot \Gamma_{f}\left(\kappa_{j}\right)}{\Gamma_{H}\left(\kappa_{j}\right)}
$$

The $\kappa$-parameters represent multiplicative deviations from the SM expectations for the different couplings. Some production/decay channels have initial/final states that can be reached through several different loops. Here, it is possible to access the relative sign of some couplings, not only the squared values. This may be particularly interesting when the interference is between fermion and boson loops, such as in the two-photon final state, where we have approximately: ${ }^{3}$

$$
\kappa_{g}=1.59 \cdot \kappa_{W}^{2}+0.07 \cdot \kappa_{t}^{2}-0.66 \cdot \kappa_{W} \kappa_{t}
$$

In order to enhance the sensitivity to particular aspects, tests can be performed under different assumptions and constraints on the coupling parameters involved.

\section{Signal Samples}

The ATLAS detector is designed to handle extremely high collision rates, while maintaining excellent identification and measurements of photons, electrons, muons and jets. A description is found elsewhere. ${ }^{4}$ Details of event selections at $13 \mathrm{TeV}$ are found in separate notes on the two photons ${ }^{5}$ and four leptons ${ }^{6}$ final states. The most important requirements on the fiducial regions are as follows: Photons are required to have pseudorapidities below $|\eta|=2.37$, excluding the region $1.37 \leq|\eta|<1.52$. Furthermore the leading and subleading photons of the Higgs candidate are required to satisfy $E_{\mathrm{T}} / m_{\gamma \gamma}>0.35$ and 0.25 respectively. The rapidity of reconstructed Higgs candidates is constrained to $|y|<2.5$. In the $4 \ell$ analysis, electrons are required to have $E_{\mathrm{T}}>7 \mathrm{GeV}$ and $|\eta|<2.47$, while muons must satisfy $p_{\mathrm{T}}>5 \mathrm{GeV}$ and $|\eta|<2.7$. Figure 3 shows resulting invariant mass distributions. 


\section{B. Stugu}

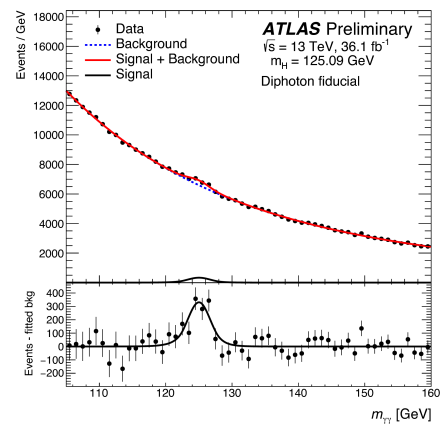

a)

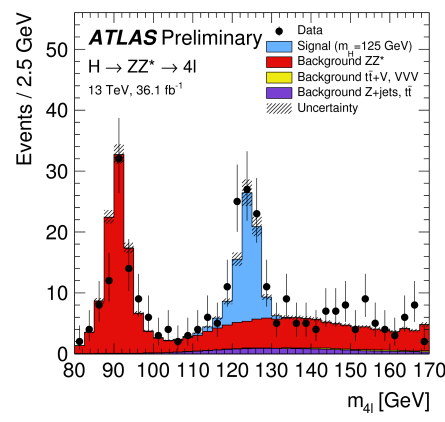

b)

Fig. 3. Invariant mass distributions of two photons a) and four leptons b) candidates from ATLAS data at $13 \mathrm{TeV}$ collected in 2015 and 2016.

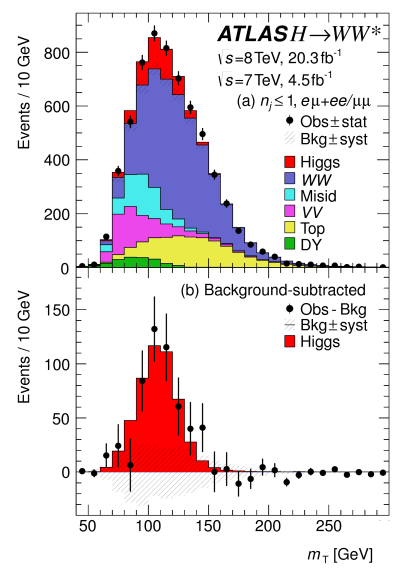

a)

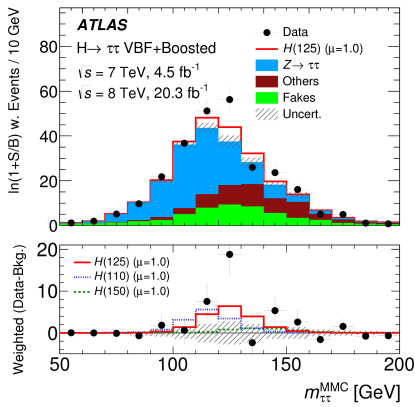

b)

Fig. 4. a): Transverse mass distributions of $H \rightarrow W^{+} W^{-} \rightarrow \ell^{+} \nu \ell^{-} \bar{\nu}$ events and b) distributions of invariant mass estimates of $H \rightarrow \tau^{+} \tau^{-}$: candidates at 7 and $8 \mathrm{TeV}$. Each event in plot b) is weighted with its signal significance.

The events can be classified into different production modes by looking for additional properties of the event: VBF candidates are found by tagging extra jets in a region extending to $|\eta|=4$, and $V H$ candidates can be found by identifying leptonic decays of the associated $W$ or $Z$ boson.

The cross section and coupling analysis at 7 and $8 \mathrm{TeV}^{7}$ include results from more final states, notably the $H \rightarrow W^{+} W^{-} \rightarrow \ell^{+} \nu \ell^{-} \bar{\nu}$ and $H \rightarrow \tau^{+} \tau^{-}$final states as shown in Fig. 4. ${ }^{8,9}$ Furthermore, all ATLAS and CMS results at 7 and $8 \mathrm{TeV}$ are combined to give an optimal set of results for all available LHC data at those collision energies. ${ }^{10}$ 


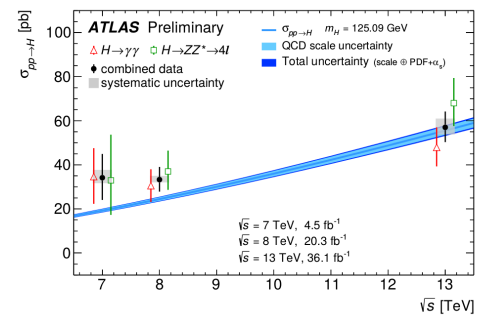

a)

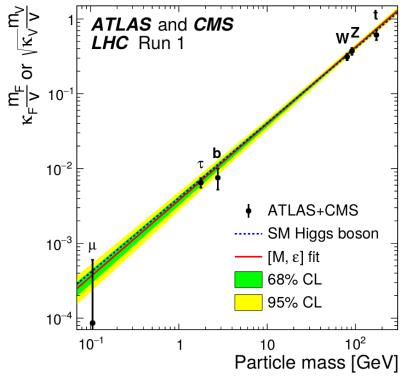

b)

Fig. 5. a) Cross sections for Higgs production as estimated with data from ATLAS. For clarity, the data points in individual decay channels are displaced along the horizontal axis. ${ }^{12}$ The banded line is represents the current best theoretical estimate. ${ }^{11} \mathrm{~b}$ ) Combined CMS and ATLAS coupling measurements using all available data at 7 and $8 \mathrm{TeV} .{ }^{10}$

\section{Results}

\subsection{Cross Sections}

Fig. 5a) summarises the Higgs production cross sections as measured by ATLAS in the $\gamma \gamma$ and $4 \ell$ final states. They are consistent with the updated SM predictions ${ }^{11}$ (superseding values used in the analyses at 7 and $8 \mathrm{TeV}^{3}$ ). The preliminary ATLAS measurement of the global signal strength at $13 \mathrm{TeV}$ is determined from the $\gamma \gamma$ and $4 \ell$ final states to be ${ }^{12}$

$$
\mu=1.09 \pm 0.12=1.09 \pm 0.09 \text { (stat.) }{ }_{-0.05}^{+0.06} \text { (exp.) }{ }_{-0.05}^{+0.06} \text { (th.) }
$$

where systematic errors are decomposed into separate contributions from experimental and theoretical uncertainties. The signal strengths can be decomposed into different production modes, as depicted in Fig. 5b)

\subsection{Couplings}

Comprehensive analyses of the couplings are already performed using data at 7 and $8 \mathrm{TeV}$ from ATLAS data alone $^{7}$ and from a combined ATLAS and CMS data. ${ }^{10}$ The universal nature of the Higgs couplings to mass is nicely depicted in Fig. 5b).

The diphoton analysis of $13 \mathrm{TeV}$ data $^{5}$ aimed at estimating the strengths of the different production modes follows the same strategy as used at 7 and $8 \mathrm{TeV}$. Here, the events are classified into 31 experimental categories with varying content of production modes (including modes with associated heavy quarks). The full set of modes is presented and discussed in the publications concerning data at 7 and 8 TeV. ${ }^{7,10}$

A likelihood method can subsequently be used to determine the most likely values of the couplings. Constraints can be imposed to study particular couplings of interest. Using the SM predictions for the two-photon branching fraction, the twophoton analysis gives estimates of the production couplings as depicted in Fig. 6a). 


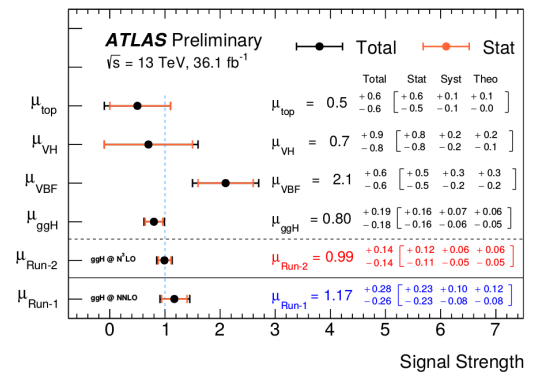

a)

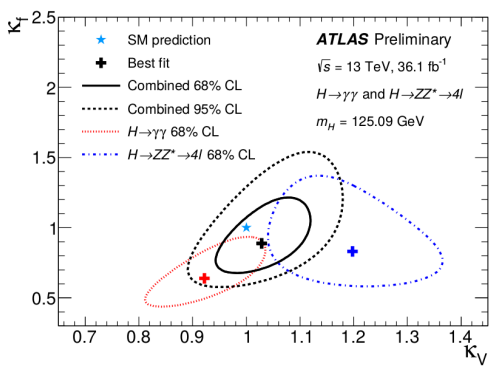

b)

Fig. 6. Estimates of Higgs signal strengths (a) and couplings (b) from data at $13 \mathrm{TeV}$.

Combining the two analyses at $13 \mathrm{TeV}$, new estimates of the Higgs couplings to fermions $\left(\kappa_{F}\right)$ and vector bosons $\left(\kappa_{V}\right)$ are performed. The ambiguity of the relative sign of the two couplings is now resolved, and it is determined that they have the same sign. Figure $6 \mathrm{~b}$ ) shows confidence contours of the estimates in the $\kappa_{F}-\kappa_{V}$ plane.

\section{Conclusion and outlook}

All estimates of Higgs production cross section and couplings are found to be consistent with the SM predictions. The precision of the estimates is starting to approach the $10 \%$ level in some single parameter fits using the data collected in 2016 at $\sqrt{s}=13 \mathrm{TeV}$. With the prospect of well above $100 \mathrm{fb}^{-1}$ per experiment for the full Run 2 dataset, very significant improvements on the precision are yet to be expected.

\section{References}

1. ATLAS Collaboration, Phys. Lett. B716 (2012) 1.

2. CMS Collaboration, Phys. Lett. B716 (2012) 30.

3. S Heinemeyer et al., Handbook of LHC Higgs Cross Sections: 3. Higgs Properties (2013), arXiv:1307.1347 [hep-ph].

4. ATLAS Collaboration, 2008 JINST 3 S08003.

5. ATLAS Collaboration, ATLAS-CONF-2017-045.

6. ATLAS Collaboration, ATLAS-CONF-2017-032.

7. ATLAS Collaboration, Eur. Phys. J. C76 (2016) 6.

8. ATLAS Collaboration, Phys. Rev. D22, 012006 (2015).

9. ATLAS Collaboration, JHEP 04 (2015) 117.

10. ATLAS and CMS Collaborations, JHEP08 (2016) 045.

11. D. de Florian et al., Handbook of LHC Higgs Cross Sections: 4. Deciphering the Nature of the Higgs Sector, (2016), arXiv: 1610.07922 [hep-ph].

12. ATLAS Collaboration, ATLAS-CONF-2017-047. 